\title{
Weltklimakonferenz in Durban: Über Inhalte einigen wir uns später
}

Die 17. Weltklimakonferenz in Durban war die längste in der Geschichte. Nach einem Verhandlungsmarathon mit teilweise heftigen Auseinandersetzungen einigten sich die Staaten darauf, bis 2015 ein globales Klimaabkommen zu erarbeiten. Dieses wird dann ab dem Jahr 2020 in Kraft treten. Es soll erstmals alle Staaten rechtlich binden, also insbesondere die USA und aufstrebende Staaten wie China, Indien und Brasilien in den internationalen Klimaschutz einbinden. Daneben wurde vereinbart, das Kyoto-Protokoll um eine weitere Verpflichtungsperiode zu verlängern. Schließlich wurden verschiedene Beschlüsse der letzten Klimakonferenzen weiterentwickelt, etwa der Grüne Klimafonds, der öffentliche und private Finanzmittel zur klimafreundlichen Entwicklung und Anpassung an den Klimawandel in Entwicklungsländern mobilisieren soll. Deutschland möchte gerne Sitz dieses Fonds werden, in den die Industrieländer ab dem Jahr 2020 jährlich 100 Mrd. US-\$ einzahlen sollen. Die Finanzierung dieser Summe bleibt aber weiter unklar und dürfte extrem schwierig werden, nicht nur in Europa mit seinen wirtschaftlichen Problemen und stark gefallenen $\mathrm{CO}_{2}$-Preisen.

Das Kyoto-Protokoll, das bisher einzig verbindliche internationale Abkommen zum Klimaschutz, ist 2005 in Kraft getreten und läuft 2012 aus. Bezüglich der Reduktionsziele unterscheidet das Kyoto-Protokoll zwischen Industrie- und Entwicklungsländern: Einige Industrieländer verpflichten sich zu Emissionsminderungen, während die Entwicklungs- und Schwellenländer ohne Reduktionsverpflichtungen bleiben. Dieser Ansatz - relativ ambitionierte Klimaziele für eine kleine Zahl von Staaten - ist aber nicht in der Lage, das Ansteigen der Durchschnittstemperatur gegenüber der vorindustriellen Zeit, wie auf dem Gipfel von Cancun beschlossen, bis zum Jahre 2100 auf $2^{\circ} \mathrm{C}$ zu begrenzen. So ist mit den USA der wichtigste Spieler auf Seiten der Industrieländer im Kyoto-Prozess außen vor. Aber auch ein Alleingang der Industrienationen würde nicht ausreichen. China hat mittlerweile die USA als größten $\mathrm{CO}_{2}$-Emittenten abgelöst. Die Schwellen- und Entwicklungsländer, neben China vor allem Brasilien und Indien, tragen immer mehr zum Klimawandel bei, beteiligen sich aber bisher nicht an dessen Verminderung. Eine kosteneffiziente internationale Klimapolitik, die Klimaschutzziele zu möglichst geringen Kosten erreicht, ist so nicht umzusetzen. Und ohne eine sinnvolle ökonomische Strategie werden die geforderten drastischen Emissionsreduktionen in wirtschaftlichen Krisenzeiten nicht zu schaffen sein. Das Kyoto-Protokoll ist daher ein Auslaufmodell. Das Bekenntnis der „Durban Plattform“ zu einem umfassenden internationalen Abkommen ab 2020 ist ein wichtiger Schritt in der langen und zähen Klimadiplomatie.

Bis 2020 bleibt aber das Kyoto-Protokoll neben den freiwilligen Zielen von Kopenhagen „the only game in town“. Die endgültige Entscheidung über eine zweite Verpflichtungsperiode des Kyoto-Protokolls (bis 2017 oder 2020) soll bei der nächsten Vertragsstaatenkonferenz in Katar 2012 fallen. Japan, Kanada und Russland haben bereits angekündigt, dass sie dann nicht mehr dabei sein wollen. Dafür werden Neuseeland und vielleicht Australien beitreten. In diesem Jahrzehnt werden daher $85 \%$ der globalen Emissionen ohne verbindliche Reglementierung sein. Die Weiterführung des Kyoto-Protokolls war die zentrale Forderung wichtiger Schwellenländer in Durban. Europa wollte dies eigentlich nicht, da in den Strukturen von Kyoto eben keine weitreichenden Fortschritte möglich sind. Nun hat man einer Verlängerung zugestimmt, um im Gegenzug zwar keine stärkeren Verpflichtungen für das nächste Jahrzehnt zu erhalten, aber zumindest die Zusage zu einem globalen

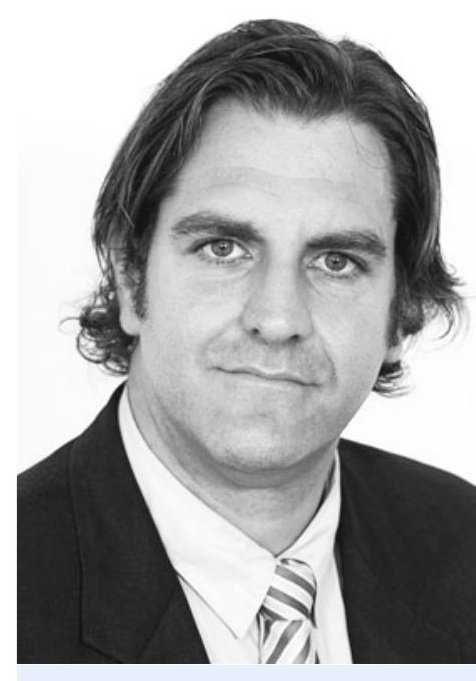

Andreas Löschel ist Professor für Umweltökonomik an der Universität Heidelberg und Leiter des Forschungsbereichs Umwelt- und Ressourcenökonomik, Umweltmanagement am Zentrum für Europäische Wirtschaftsforschung (ZEW) in Mannheim. 
Abkommen ab 2020. Ohne das Engagement Europas - im Schulterschluss mit den vom Klimawandel am stärksten betroffenen Staaten - wäre Durban gescheitert. Wenigen Ländern liegt der Klimaschutz nämlich wirklich am Herzen. Die Widerstände der USA oder Kanadas auf der einen Seite und Indiens und zum Teil Chinas auf der der anderen Seite waren bei diesen Verhandlungen wieder immens. Die Weiterführung des Kyoto-Protokolls wird aber keine großen Auswirkungen für Europa haben: Die Zielsetzung wird sich an den EU-2020-Zielen orientieren, d.h. Europa macht das, was es sowieso vorhatte zu tun.

Jetzt gibt es also einen Fahrplan und einen Termin für ein umfassendes Abkommen. Der Fahrplan bis 2015 ist allerdings nicht verbindlich und auch die Ausgestaltung des Abkommens ist noch unklar. Es wird eben nicht von einem rechtlich bindenden Abkommen gesprochen, sondern insbesondere auf Druck der Schwellenländer von einem Verfahren mit Rechtskraft („outcome with legal force“). Über die Höhe der zukünftigen Verpflichtungen wurde gar nichts gesagt. Man hat sich geeinigt, sich später zu einigen. Deshalb wird sich auch erst in den kommenden Jahren zeigen, in welchem Umfang die Forderungen nach Effektivität, Fairness und Effizienz des globalen Abkommens eingelöst werden können. Die bekannten grundsätzlichen ökonomischen Anreizprobleme im internationalen Klimaschutz werden auch für dieses zukünftige Abkommen gelten. Interessanterweise finden sich in den Beschlüssen von Durban kaum noch Verweise auf die Dichotomie von Industrie- und Entwicklungsländern oder auf Gerechtigkeitsvorstellungen. Aber diese werden bei der konkreten Ausgestaltung sehr schnell wieder in den Vordergrund treten.

Während die Stringenz des Klimaziels für die Akzeptanz des zukünftigen Abkommens bedeutsam ist, werden sich die Verhandlungen insbesondere auf die Verteilung der Belastungen konzentrieren. Die zentralen Bestimmungsfaktoren für das Handeln von Akteuren sind dabei die ökonomischen Kosten und Nutzen eines Verhandlungsvorschlags. Da die Kosten und Nutzen von Emissionsreduktionen regional sehr unterschiedlich sind, erfordern Kooperationen in internationalen Klimaverhandlungen auch, dass die Lastenverteilung als gerecht oder fair empfunden wird. Allerdings ist dieser faire Anteil meistens umstritten, da mehrere begründbare Fairnessnormen bestehen. Die Klimarahmenkonvention bekennt sich zum Prinzip der „gemeinsamen aber differenzierten Verantwortung“. Im Rahmen der internationalen Klimaverhandlungen werden aber verschiedene andere Gerechtigkeitsprinzipien vorgebracht, z.B. der egalitaristische Ansatz (gleichmäßige Verteilung der Emissionen auf die einzelnen Individuen), das Verursacherprinzip (derjenige muss handeln, der den Schaden verursacht und zu verantworten hat), das Leistungsfähigkeitsprinzip (derjenige muss handeln, der dazu wirtschaftlich in der Lage ist) oder der Souveränitäts-Ansatz (gleichmäßige Reduktion ausgehend vom Status quo der Emissionsverteilung).

Wir konnten kürzlich mit Hilfe einer Befragung unter Akteuren in der Klimapolitik und Modellrechnungen zeigen, dass die wahrgenommenen Positionen zu bestimmten Gerechtigkeitsprinzipien der verschiedenen Regionen sich weitgehend mit deren materiellem Eigeninteresse decken: je höher die durch ein Gerechtigkeitskriterium implizierten Kosten, umso niedriger die Unterstützung dieser Position durch eine Region. Die zu erwartende Diskussion über die Fairness eines Nachfolgeabkommens dürfte daher das Kooperationsproblem eben nicht lösen. Hierzu müssen Kosten und Nutzen für die Teilnahme an einem internationalen Abkommen

Andreas Löschel

Universität Heidelberg, ZEW loeschel@zew.de für die Staaten verbessert werden, um ökonomische Anreize zu setzen. Durch das Aufbrechen der alten Verhandlungspositionen könnte auch hier die Konferenz in Durban ein wichtiger Schritt gewesen sein. 